\title{
Synthesis of rigidified flavin-guanidinium ion conjugates and investigation of their photocatalytic properties
}

\author{
Harald Schmaderer, Mouchumi Bhuyan and Burkhard König*
}

\author{
Full Research Paper \\ Address: \\ Institute of Organic Chemisty, University of Regensburg, \\ Universitätsstr. 31, D-93040 Regensburg, Germany \\ Email: \\ Burkhard König * - burkhard.koenig@chemie.uni-regensburg.de \\ * Corresponding author \\ Keywords: \\ flavin; guanidine; Kemp's acid; photocatalysis; template
}

Open Access

\author{
Beilstein Journal of Organic Chemistry 2009, 5, No. 26 \\ doi:10.3762/bjoc.5.26 \\ Received: 27 February 2009 \\ Accepted: 07 May 2009 \\ Published: 28 May 2009 \\ Associate Editor: J. Murphy \\ (c) 2009 Schmaderer et al; licensee Beilstein-Institut. \\ License and terms: see end of document.
}

\begin{abstract}
Flavin chromophores can mediate redox reactions upon irradiation by blue light. In an attempt to increase their catalytic efficacy, flavin derivatives bearing a guanidinium ion as oxoanion binding site were prepared. Chromophore and substrate binding site are linked by a rigid Kemp's acid structure. The molecular structure of the new flavins was confirmed by an X-ray structure analysis and their photocatalytic activity was investigated in benzyl ester cleavage, nitroarene reduction and a Diels-Alder reaction. The modified flavins photocatalyze the reactions, but the introduced substrate binding site does not enhance their performance.
\end{abstract}

\section{Introduction}

Flavins are redox-active chromophores [1-6] and represent one of the most abundant classes of natural enzyme co-factors [7-9]. Recently, the photo redox properties of flavins have been used to catalyze chemical reactions [10-30]. A general drawback of photochemical processes in homogeneous solution is the limited preorganization of the reactants and the chromophore, which may lead to low selectivities and slow conversions in diffusion controlled reactions. To overcome this problem, Kemp's acid [31] derivatives have been used as sterically defined templates enhancing the efficiency and selectivity of photoreactions [32-43]. Flavins with geometrically defined substrate binding sites have not been reported so far and we expected that the close vicinity of substrate and flavin should enhance the rate of photoinduced electron transfer processes, which strongly depend on distance [44]. We present here the synthesis of geometrically defined flavin-guanidinium ion conjugates based on a Kemp's acid skeleton (Scheme 1). The guanidinium moiety should serve as a hydrogen bonding site for oxoanions or carbonyl groups [45-49]. The structure of the new flavins was determined in solid state and in solution and their photocatalytic properties were tested.

\section{Results and Discussion Synthesis}

The synthesis of the potential photocatalysts $\mathbf{1}$ and $\mathbf{2}$, consisting of the flavin chromophore, the guanidinium substrate binding 


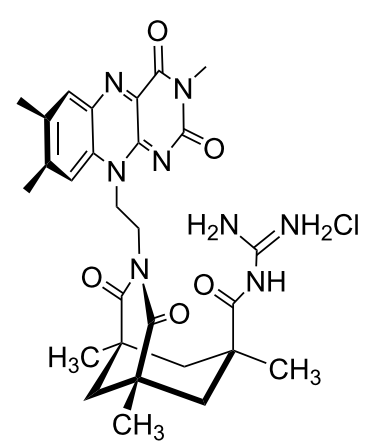

1

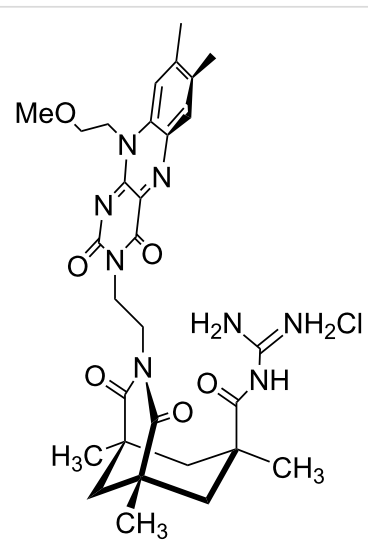

2<smiles></smiles>

3

Scheme 1: Flavin-guanidinium ion conjugates $\mathbf{1}$ and $\mathbf{2}$ and tetraacetyl riboflavin (3).

site and a Kemp's acid derived rigid linker, starts from Kemp's acid anhydride (5) [50-52]. The anhydride 5 was allowed to react with previously prepared flavins 4 and 8 [21] in the presence of DMAP as catalyst. The amide formation of the carboxyl group with Boc-protected guanidine was achieved using standard peptide coupling conditions. Boc-deprotection with hydrogen chloride in diethyl ether yielded the guanidinium chloride salts $\mathbf{1}$ and $\mathbf{2}$ (Scheme 2). The guanidinium salts are soluble in water and methanol, but also in chloroform and acetonitrile.

\section{Structural investigations}

The structure of compounds $\mathbf{1}, \mathbf{2}, \mathbf{6}$, and $\mathbf{9}$ was examined in the solid state and in solution. Figure 1 shows the X-ray crystal

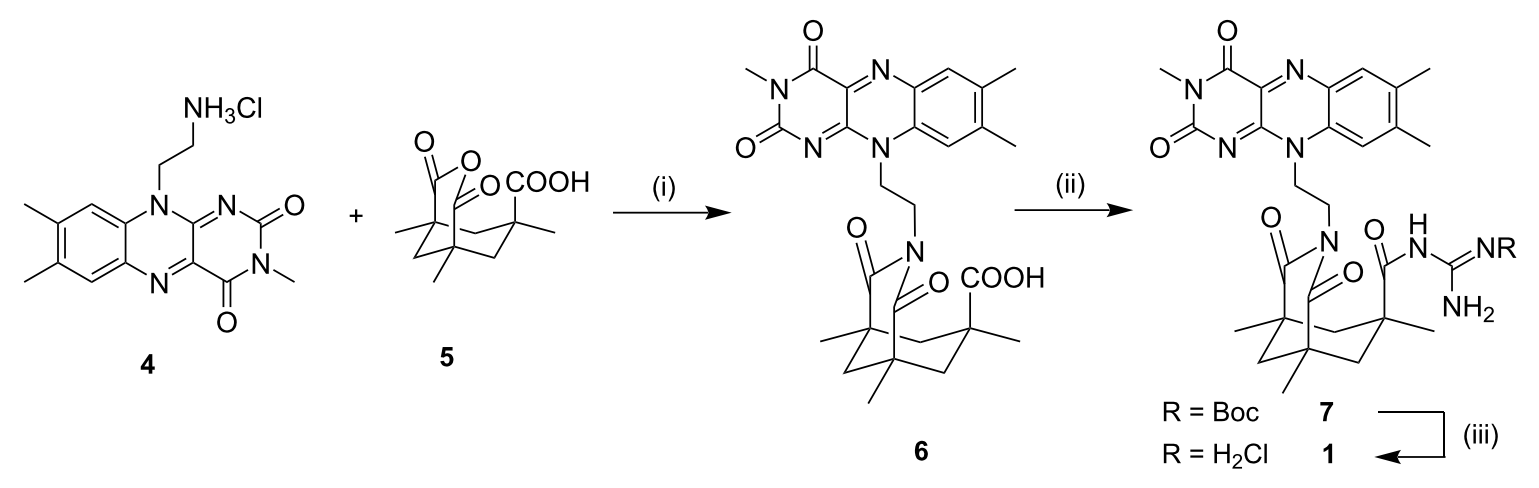

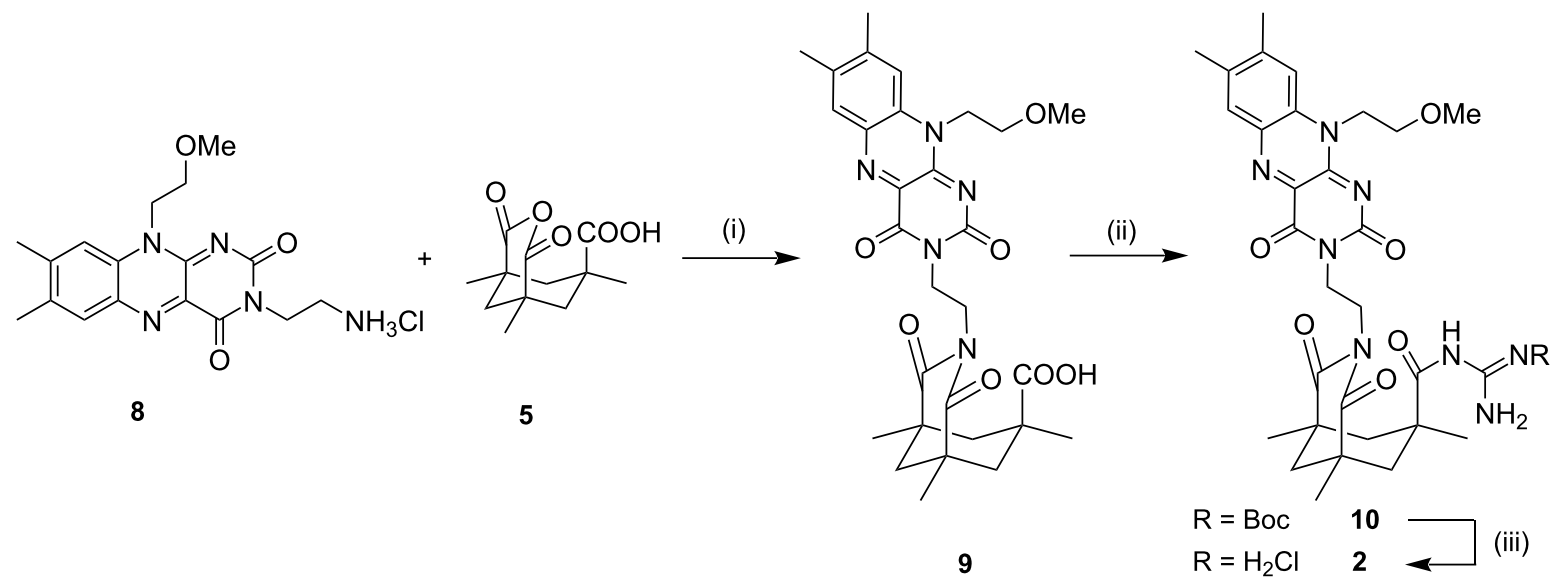

Scheme 2: Synthesis of flavins 1 and 2. Conditions: (i) DMAP, $\mathrm{H}_{2} \mathrm{O}, \Delta, 20 \mathrm{~h}, 71-78 \%$, (ii) $\mathrm{HOBt}$, EDC, NEt( $\left.\mathrm{Pr}\right)_{2}$, mono-Boc guanidine, $\mathrm{CH}_{2} \mathrm{Cl}_{2}, \mathrm{rt}, 20$ h, 58-82\%, (iii) $\mathrm{HCl} / \mathrm{Et}_{2} \mathrm{O}, \mathrm{CH}_{2} \mathrm{Cl}_{2} / \mathrm{CHCl}_{3}$, rt, 24 h, 83-90\%. 

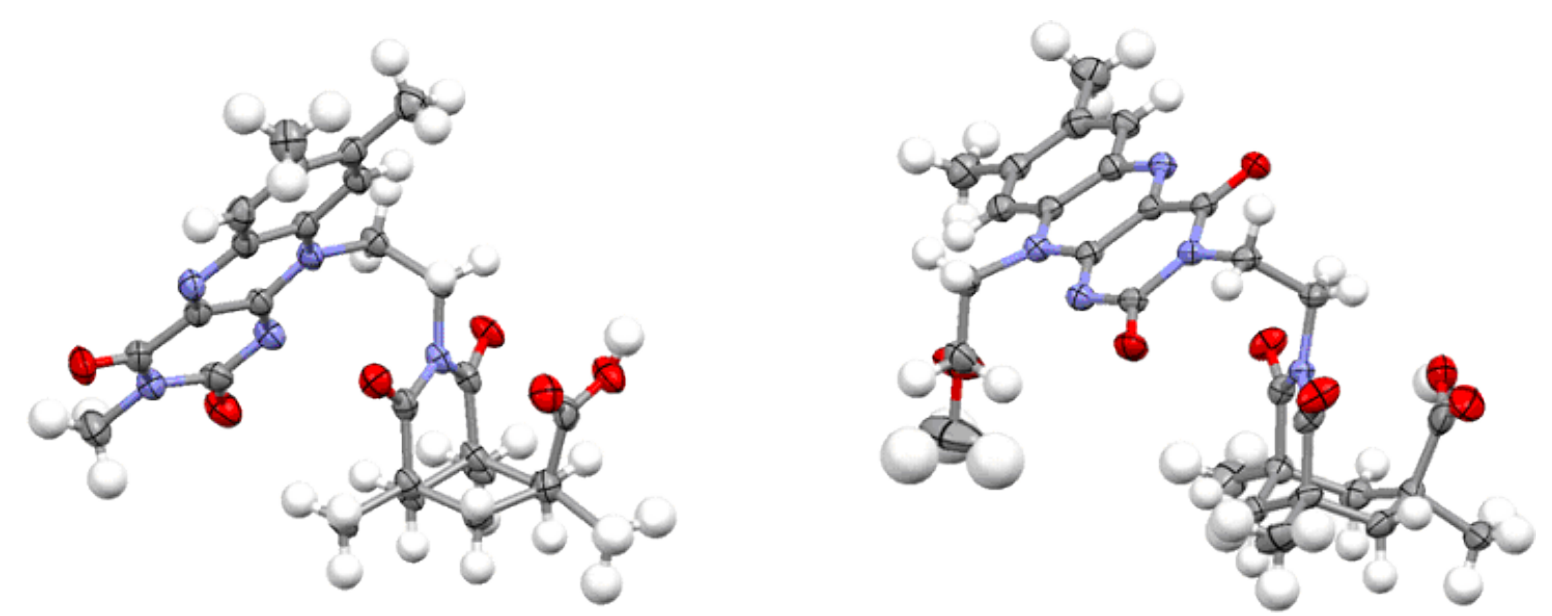

Figure 1: X-ray crystal structures of the flavin-Kemp's acids 6 (left) and 9 (right).

structures of $\mathbf{6}$ and $\mathbf{9}$. The planar flavin chromophore is turned outward relative to the Kemp's acid. Intermolecular $\pi$ - $\pi$-interactions between the flavin heteroarenes are observed.

The structure of compound $\mathbf{1}$ in the solid state (Figure 2) shows an almost identical orientation of the flavin group to that of the acid 6. The acyl guanidinium ion group is almost planar and in a parallel orientation relative to the Kemp's acid imide group.

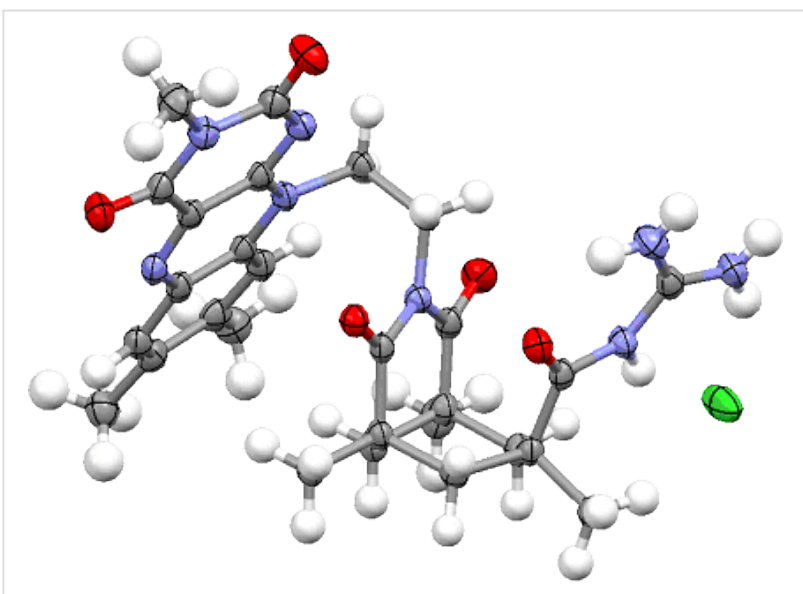

Figure 2: Structure of compound 1 in the solid state.

2-D NMR spectra of compounds $\mathbf{1}$ and $\mathbf{2}$ revealed several NOE contacts, but the flexibility of the molecule did not allow the determination of preferred conformations.

The most stable conformer of compound $\mathbf{1}$ in the gas phase was determined by computational methods (semi-empirical AM1, Spartan program package, Figure 3, see also Supporting Information File 1) [53]. In this structure the flavin is turned towards the guanidinium ion forming a hydrogen bond between the flavin carbonyl oxygen atom and the guanidinium moiety (distance $\sim 2.1 \AA$ ). However, simple gas phase calculations overestimate the effect of hydrogen bonds [54-58] and in solution the flavin chromophore is expected to rotate freely around the $\mathrm{C}-\mathrm{C}$ single bonds of the ethane linker.

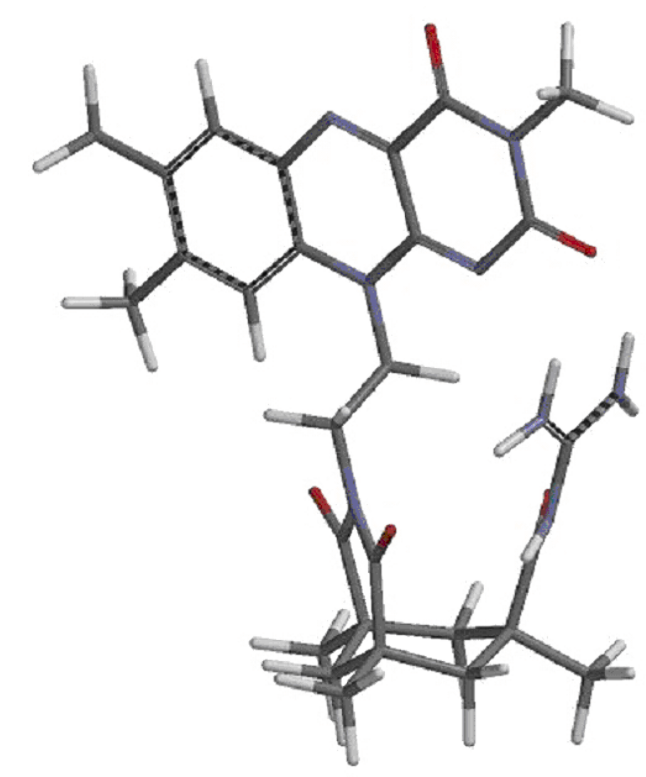

Figure 3: Calculated lowest energy conformation of 1 in the gas phase (AM1, Spartan program package).

\section{Photocatalytic reactions}

Compounds $\mathbf{1}$ and $\mathbf{2}$ were tested as photocatalysts in three different reactions and their performance was compared to tetraacetyl riboflavin 3 or compound 8. Dibenzyl phosphate esters are oxidatively cleaved by blue light irradiation (440 nm) in the presence of compounds $\mathbf{1}$ and $\mathbf{2}$ (Scheme 3). The acceleration of the reaction in acetonitrile by $\mathbf{1}$ and $\mathbf{2}$, bearing a guan- 
idinium ion binding site with phosphate affinity, is significantly larger (Table 1, entries 1+2) in comparison to the ammonium salt 8 (entry 3). In water, however, the accelerating effect is not observed (entries 5-8). The presence of the photocatalyst is essential in all cases, as the non-catalyzed hydrolysis is slow under the reaction conditions ( $<5 \%$ conversion).

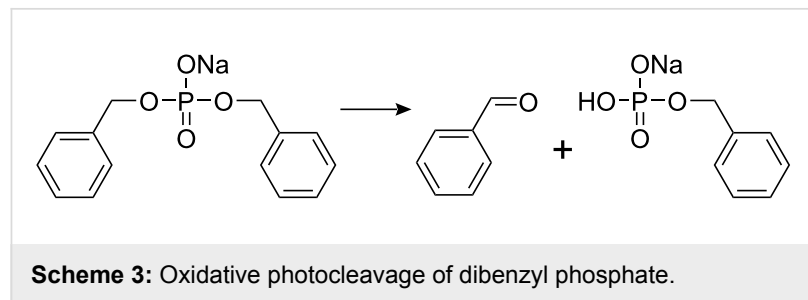

\begin{tabular}{lllll}
\hline \multicolumn{5}{l}{ Table 1: Oxidative photocleavage of dibenzyl phosphate. } \\
\begin{tabular}{lllll} 
Entry & Catalyst & Solvent & $\mathrm{t}(\mathrm{h})$ & Conversion (\%) \\
\hline $1^{\mathrm{a}}$ & $\mathbf{1}$ & $\mathrm{MeCN}-d_{3}$ & 4 & 53 \\
$2^{\mathrm{a}}$ & $\mathbf{2}$ & $\mathrm{MeCN}-d_{3}$ & 4 & 58 \\
$3^{\mathrm{a}}$ & $\mathbf{8}$ & $\mathrm{MeCN}-d_{3}$ & 4 & 12 \\
$4^{\mathrm{a}}$ & - & $\mathrm{MeCN}-d_{3}$ & 4 & $<5$ \\
\hline 5 & $\mathbf{1}$ & $\mathrm{D}_{2} \mathrm{O}$ & 2 & 44 \\
6 & 2 & $\mathrm{D}_{2} \mathrm{O}$ & 2 & 15 \\
7 & $\mathbf{8}$ & $\mathrm{D}_{2} \mathrm{O}$ & 2 & 50 \\
8 & - & $\mathrm{D}_{2} \mathrm{O}$ & 2 & $<5$ \\
\hline
\end{tabular}
\end{tabular}

Conditions: $V=1 \mathrm{~mL}$, dibenzyl phosphate $10^{-2} \mathrm{M}$, catalyst $20 \mathrm{~mol} \%, 40$ ${ }^{\circ} \mathrm{C}$, LED (440 nm). aDibenzyl phosphate ester was neutralized previous to the reaction.

In the presence of sacrificial electron donor substrates, such as aliphatic amines, flavins can photoreduce nitro arenes to anilines under blue light irradiation (Scheme 4). 4-Nitrophenyl phosphate was used as a substrate for photoreduction in water and in acetonitrile. The results summarized in Table 2 show that $10 \mathrm{~mol} \%$ of flavin 2 , the same amount of tetraacetyl riboflavin (3) or compound $\mathbf{8}$ catalyze the photoreaction equally well. The guanidinium ion binding site of $\mathbf{1}$ and $\mathbf{2}$ does not lead to a more effective conversion.

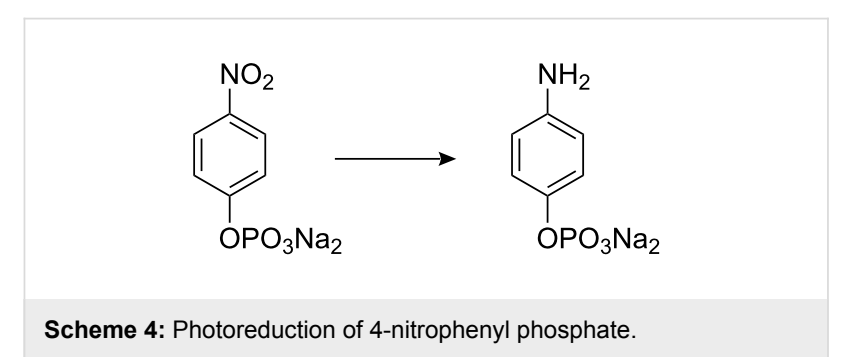

The intermolecular interaction of the guanidinium ion binding site of compound $\mathbf{2}$ with phosphate ester anions and dianions was probed by UV/vis and emission spectroscopy in acetonitrile and buffered aqueous solution. The emission intensity of the chromophore of $\mathbf{2}$ decreased slightly in the presence of the anions in acetonitrile indicating a weak interaction. In aqueous solution the presence of the anions did not induce significant changes of the emission properties suggesting affinity constants smaller than $10^{3} \mathrm{~L} / \mathrm{mol}$.

\begin{tabular}{|lllc}
\hline \multicolumn{3}{|l}{ Table 2: Results of nitrobenzene photoreduction. } \\
\hline \multicolumn{1}{|l}{ Entry } & Catalyst & Solvent & Conversion (\%) \\
\hline 1 & $\mathbf{1}$ & $\mathrm{H}_{2} \mathrm{O}$ & 36 \\
2 & $\mathbf{2}$ & $\mathrm{H}_{2} \mathrm{O}$ & 72 \\
3 & $\mathbf{2}$ & $\mathrm{H}_{2} \mathrm{O}^{\mathrm{a}}$ & 73 \\
4 & $\mathbf{3}$ & $\mathrm{H}_{2} \mathrm{O}^{\mathrm{a}}$ & 89 \\
5 & $\mathbf{8}$ & $\mathrm{H}_{2} \mathrm{O}$ & 79 \\
\hline $6^{\mathrm{b}}$ & $\mathbf{1}^{\mathrm{c}}$ & $\mathrm{MeCN}$ & 15 \\
$7^{\mathrm{b}}$ & $\mathbf{2}$ & $\mathrm{MeCN}$ & 55 \\
$8^{\mathrm{b}}$ & $\mathbf{3}$ & $\mathrm{MeCN}$ & 81 \\
$9^{\mathrm{b}}$ & $\mathbf{8}$ & $\mathrm{MeCN}$ & 59 \\
\hline
\end{tabular}

Conditions: $\mathrm{V}=5 \mathrm{~mL}$, nitrobenzene $10^{-2} \mathrm{M}$, catalyst $10 \mathrm{~mol} \%$, $\mathrm{N}\left(\mathrm{CH}_{2} \mathrm{CH}_{2} \mathrm{OH}\right)_{3} 10$ equiv, $\mathrm{t}=4 \mathrm{~h}, 40^{\circ} \mathrm{C}$, LED (440 nm), UV-lamp (370 nm). ${ }^{1} 10 \%$ DMSO added to increase solubility. ${ }^{\text {b}} 4-$ Nitrophenyl phosphate was neutralized previous to the reaction. ${ }^{\mathrm{C}}$ The catalyst is barely soluble in $\mathrm{MeCN}$, which explains the lower conversion in this case.

Photo Diels-Alder reactions in the presence of a sensitizer and light have been described [59-64]. Therefore flavins 1 and 2 were tested as catalyst for the cycloaddition of maleimide to anthracene in toluene (Scheme 5). Table 3 summarizes the results. A significantly higher yield of the cycloaddition product was obtained after $8 \mathrm{~h}$ at $40{ }^{\circ} \mathrm{C}$ in the presence of compound 2 (entry 3), if compared to the control reaction (entry 6). Upon irradiation with blue light the yield after $8 \mathrm{~h}$ reaction time increased further (entry 2) and was significantly higher as in the absence of a photocatalyst (entry 5). However, a comparison with tetraacetyl riboflavin (3) under identical reaction conditions showed an even more pronounced acceleration of the reaction (entry 4). Blue light irradiated flavins accelerate the anthracene maleimide cycloaddition significantly, but flavins $\mathbf{1}$ and $\mathbf{2}$

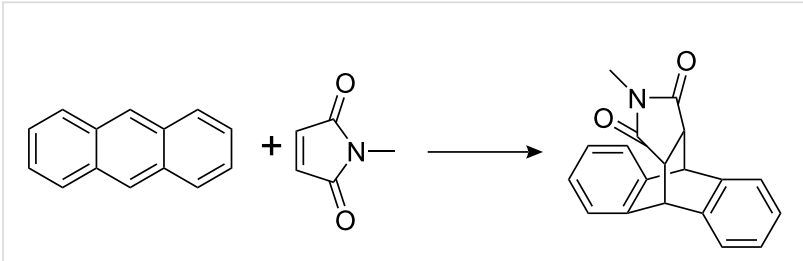

Scheme 5: Photo Diels-Alder-reaction of anthracene with N-methylmaleinimide. 
do not provide additional benefit if compared to tetraacetyl flavin 3.

\begin{tabular}{|llllll}
\multicolumn{6}{c}{ Table 3: Results of photoinduced Diels-Alder-reaction. } \\
\begin{tabular}{|llllll} 
Entry & Catalyst & hv & Yield (\%) & TON & TOF $\left(\mathrm{h}^{-1}\right)$ \\
\hline 1 & 1 & + & 45 & 22.5 & 2.8 \\
2 & 2 & + & 85 & 42.5 & 5.3 \\
3 & 2 & - & 59 & 28.5 & 3.6 \\
4 & 3 & + & 100 & 50 & 6.3 \\
5 & - & + & 30 & - & - \\
6 & - & - & 9 & - & - \\
$7^{\mathrm{a}}$ & - & - & 100 & - & - \\
\hline
\end{tabular}
\end{tabular}

Conditions: Toluene, $\mathrm{V}=1.2 \mathrm{~mL}$, anthracene $33 \times 10^{-3} \mathrm{M}$, maleinimide 2.5 equiv, catalyst $2 \mathrm{~mol} \%, \mathrm{t}=8 \mathrm{~h}, 40^{\circ} \mathrm{C}$, LED $(440 \mathrm{~nm})$. ${ }^{a}$ Anthracene $500 \mu \mathrm{mol}$, methyl maleinimide $1.25 \mathrm{mmol}$, toluene $10 \mathrm{~mL}, 100^{\circ} \mathrm{C}, 16 \mathrm{~h}$.

\section{Conclusion}

We have prepared new flavin derivatives that bear an acyl guanidinium group, which is linked to the chromophore via a rigid Kemp's acid spacer. The connectivity and expected relative geometry of 1 and of the carboxylic acids 6 and 9 was confirmed by X-ray structure analysis. Guanidinium cations are known to bind oxoanions, such as phosphates, via hydrogen bonds. Therefore a benefit to the photocatalytic activity of $\mathbf{1}$ and 2 was expected, as the binding site could keep reaction substrates in close proximity to the redox active chromophore, facilitating photoinduced electron transfer processes. Initial exemplary photocatalytic experiments showed that flavin-derivatives $\mathbf{1}$ and $\mathbf{2}$ catalyze oxidative benzyl ether cleavage, nitro arene reductions and Diels-Alder reactions. However, no significant gain in photocatalytic performance by the guanidinium ion substrate binding site was observed in comparison to flavins lacking the binding site and the rigid Kemp's acid skeleton. The primary interaction between the aromatic substrates and the heteroaromatic flavin chromophore seems to dominate the formation of the substrate-catalyst aggregate. Hydrogen bonds between the substrate and the acylguanidinium group are not decisive for their interaction. The rigidity of the Kemp's triacid skeleton is not effectively transferred in $\mathbf{1}$ and $\mathbf{2}$ to the relative flavin-guanidinium ion orientation, which is due to the flexible ethane linker between imide and flavin. Derivatives with a more constrained conformation of the flavin chromophore and the substrate binding sites may lead to chemical photocatalysts with better performance.

\section{Experimental General}

The flavin salts 4 [10-(2-aminoethyl)-3,7,8trimethylbenzo[g]pteridine-2,4(3H,10H)-dione] and 8 [3-(2- aminoethyl)-10-(2-methoxyethyl)-7,8-dimethylbenzo[g]pteridine-2,4(3H,10H)-dione], Kemp's acid anhydride 5 (1,5,7trimethyl-2,4-dioxo-3-oxa-bicyclo[3.3.1]nonane-7-carboxylic acid) and mono Boc-protected guanidine were prepared by known methods [21,50-52]. All other chemicals were purchased from commercial suppliers, checked by ${ }^{1} \mathrm{H}$ NMR spectrometry and then used as received. Solvents were distilled before use. Flash column chromatography was carried out on silica gel 35-70 $\mu \mathrm{m}, 60 \AA$ from Acros. NMR spectra were recorded at a Bruker Avance 300 spectrometer $(300 \mathrm{MHz})$ or at a Bruker Avance 600 spectrometer $(600 \mathrm{MHz})$. Electrospray ionisation (ES-MS) mass spectra were measured on ThermoQuest Finnigan TSQ 7000 spectrometer. High resolution mass spectrometry (HRMS) was measured on ThermoQuest Finnigan MAT 95 spectrometer. Melting points were measured on a Büchi SMP-20 apparatus and are not corrected. IR spectra were measured on Biorad Spectrometer Excalibur FTS 3000. UV/Vis spectra were recorded at Varian Cary 50 Bio UV/VIS spectrometer against air. Fluorescence spectra were recorded at Varian Cary Eclipse.

\section{1,5,7-Trimethyl-2,4-dioxo-3-[2-(3,7,8- trimethyl-2,4-dioxo-3,4-dihydrobenzo[ $g$ ]pter- idin-10(2H)-yl)ethyl]-3- azabicyclo[3.3.1]nonane-7-carboxylic acid; Flavin-Kemp's acid 6}

DMAP (230 mg, $1.9 \mathrm{mmol}$ ) and Kemp's acid anhydride (5) (180 mg, $750 \mu \mathrm{mol}$ ) were added successively to a solution of flavin salt $4(250 \mathrm{mg}, 750 \mu \mathrm{mol})$ in water $(22 \mathrm{~mL})$ and the solution was refluxed for $20 \mathrm{~h}$. After cooling, the mixture was brought to $\mathrm{pH} 1$ with hydrochloric acid $(5 \mathrm{M})$, and the precipitating orange product was collected by filtration. As thin layer chromatography showed considerable amounts of the product in the filtrate, it was concentrated and purified by flash column chromatography $\left(\mathrm{CHCl}_{3}: \mathrm{MeOH}-15: 1\right)$, to yield another portion of orange solid. Yield: $302 \mathrm{mg}, 580 \mu \mathrm{mol}, 78 \%$, orange solid; $R_{\mathrm{f}}=0.2\left(\mathrm{CHCl}_{3}: \mathrm{MeOH}-10: 1\right) ; \mathrm{mp} 292{ }^{\circ} \mathrm{C}$ (decomp.); ${ }^{1} \mathrm{H} \mathrm{NMR}\left(\mathrm{DMSO}-d_{6}\right) \delta=0.87$ (s, $6 \mathrm{H}, 2 \times$ Kemps-CH$), 1.00$ (s, $3 \mathrm{H}$, Kemps-CH$), 1.07-1.26\left(\mathrm{~m}, 3 \mathrm{H}, H_{\mathrm{ax}}\right), 2.20-2.24(\mathrm{~m}, 3$ $\left.\mathrm{H}, H_{\mathrm{eq}}\right), 2.41\left(\mathrm{~s}, 3 \mathrm{H}, \mathrm{Ar}-\mathrm{CH}_{3}\right), 2.50\left(\mathrm{~s}, 3 \mathrm{H}, \mathrm{Ar}-\mathrm{CH}_{3}\right.$, hidden by DMSO), 3.28 (s, $3 \mathrm{H}, \mathrm{N}-\mathrm{CH}_{3}$ ), 3.90 (s, $2 \mathrm{H}, \mathrm{CH}_{2}$ ), 4.87 (s, $2 \mathrm{H}$, $\mathrm{CH}_{2}$ ), 7.77 (s, $\left.1 \mathrm{H}, \mathrm{Ar}-H\right), 7.98$ (s, $\left.1 \mathrm{H}, \mathrm{Ar}-H\right), 12.24$ (br s, $1 \mathrm{H}$, $\mathrm{COOH}) ;{ }^{13} \mathrm{C} \mathrm{NMR}\left(\mathrm{DMSO}-d_{6}\right) \delta=18.8\left(\mathrm{Ar}-\mathrm{CH}_{3}\right), 20.9(\mathrm{Ar}-$ $\left.\mathrm{CH}_{3}\right), 24.7\left(2 \times \mathrm{CH}_{3}\right), 28.0\left(\mathrm{~N}-\mathrm{CH}_{3}\right), 29.7\left(\mathrm{CH}_{3}\right), 37.3$ $\left(\mathrm{N}-\mathrm{CH}_{2}\right), 39.4\left(2 \times C_{q u}\right), 40.9\left(2 \times \mathrm{CH}_{2}\right), 41.1\left(C_{q u}\right), 42.1$ $\left(\mathrm{CH}_{2}\right), 43.0\left(\mathrm{~N}-\mathrm{CH}_{2}\right), 116.0(C-9), 131.1(C-9 \mathrm{a}), 131.3(C-6)$, 134.2 (C-5a), 135.5 (C-4a), 136.2 (C-7), 146.9 (C-8), 149.4 (C-10a), 154.9 (C-2), $159.5(C-4), 176.2$ (2 × CO), 176.5 (CO); ES-MS $m / z(\%): 522.4(100)[\mathrm{M}+\mathrm{H}]^{+}$; HRMS-EI $m / z$ : calcd for $\mathrm{C}_{23} \mathrm{H}_{32} \mathrm{~N}_{5} \mathrm{O}_{6}[\mathrm{M}+\mathrm{H}]^{+}:$522.2353; found: $522.2342[\Delta 2.03$ 
ppm]; IR (ATR): $v=1717,1649,1583,1545,1451,1250$, 1193, 1096, 1053, 970, $756 \mathrm{~cm}^{-1}$.

\section{3-\{2-[10-(2-Methoxyethyl)-7,8-dimethyl-2,4- dioxobenzo[g]pteridin-3(2H,4H,10H)-yl]ethyl\}-} 1,5,7-trimethyl-2,4-dioxo-3azabicyclo[3.3.1]nonane-7-carboxylic acid; Flavin-Kemp's acid 9

DMAP (460 mg, $2.8 \mathrm{mmol}$ ) and Kemp's anhydride (5) (370 $\mathrm{mg}, 1.54 \mathrm{mmol})$ were added successively to a solution of flavin salt $8(570 \mathrm{mg}, 1.50 \mathrm{mmol})$ in water $(50 \mathrm{~mL})$ and the solution was refluxed for $20 \mathrm{~h}$. After cooling, the mixture was brought to $\mathrm{pH} 1$ with hydrochloric acid $(5 \mathrm{M})$, and the precipitating dark orange product was collected by filtration. As thin layer chromatography showed considerable amounts of the product in the filtrate, it was concentrated and purified by flash column chromatography $\left(\mathrm{CHCl}_{3}: \mathrm{MeOH}-15: 1\right)$, to yield another portion of orange solid. Yield: $597 \mathrm{mg}, 1.06 \mathrm{mmol}, 71 \%$, orange solid; $R_{\mathrm{f}}$ $=0.2\left(\mathrm{CHCl}_{3}: \mathrm{MeOH}-10: 1\right) ; \mathrm{mp} 305{ }^{\circ} \mathrm{C}($ decomp. $) ;{ }^{1} \mathrm{H}$ NMR $\left(\mathrm{DMSO}-d_{6}\right) \delta=0.97\left(\mathrm{~s}, 6 \mathrm{H}, 2 \times \mathrm{Kemps}^{-} \mathrm{CH}_{3}\right), 1.04(\mathrm{~s}, 3 \mathrm{H}$, Kemps- $\mathrm{CH}_{3}$ ), 1.15 (d, $\left.J=13.72 \mathrm{~Hz}, 2 \mathrm{H}, H_{\mathrm{ax}}\right), 1.32$ (d, $J=$ $\left.12.62 \mathrm{~Hz}, 1 \mathrm{H}, H_{\mathrm{ax}}\right), 1.82\left(\mathrm{~d}, J=12.62 \mathrm{~Hz}, 1 \mathrm{H}, H_{\mathrm{eq}}\right), 2.25(\mathrm{~d}, J$ $\left.=13.17 \mathrm{~Hz}, 2 \mathrm{H}, H_{\mathrm{eq}}\right), 2.41\left(\mathrm{~s}, 3 \mathrm{H}, \mathrm{Ar}-\mathrm{CH}_{3}\right), 2.50(\mathrm{~s}, 3 \mathrm{H}$, $\mathrm{Ar}-\mathrm{CH}_{3}$, hidden by DMSO), 3.22 (s, $\left.3 \mathrm{H}, \mathrm{O}-\mathrm{CH}_{3}\right), 3.74-3.77$ $\left(\mathrm{m}, 4 \mathrm{H}, 2 \times \mathrm{CH}_{2}\right), 4.06-4.07\left(\mathrm{~m}, 2 \mathrm{H}, \mathrm{N}-\mathrm{CH}_{2}\right), 4.83(\operatorname{tr}, J=$ $\left.5.35 \mathrm{~Hz}, 2 \mathrm{H}, \mathrm{O}-\mathrm{CH}_{2}\right), 7.91$ (s, $\left.1 \mathrm{H}, \mathrm{Ar}-H\right), 7.98$ (s, $\left.1 \mathrm{H}, \mathrm{Ar}-H\right)$, 12.25 (br s, $1 \mathrm{H}, \mathrm{COOH}) ;{ }^{13} \mathrm{C}$ NMR (DMSO- $\left.d_{6}\right) \delta=18.8(\mathrm{Ar}-$ $\left.\mathrm{CH}_{3}\right), 20.8\left(\mathrm{Ar}-\mathrm{CH}_{3}\right), 25.0\left(\mathrm{CH}_{3}\right), 29.9\left(\mathrm{CH}_{3}\right), 37.4,41.0,41.6$ and $43.1\left(\mathrm{C}_{q u}\right.$ and $\left.\mathrm{CH}_{2}\right), 44.0\left(10-\mathrm{N}-\mathrm{CH}_{2}\right), 58.5\left(\mathrm{O}-\mathrm{CH}_{3}\right), 68.3$ $\left(\mathrm{O}-\mathrm{CH}_{2}\right), 116.9$ (C9), 130.9 (C6), 131.5 (C9a), 134.0 (C5a), 135.6 (C4a), 136.3 (C7), 147.0 (C8), 148.6 (C10a), 155.0 (C2), 159.7 (C4), 176.1 und $176.6(\mathrm{COOH}$ und $C \mathrm{ONH})$; ES-MS $m / z$ (\%): 566.3 (100) $[\mathrm{M}+\mathrm{H}]^{+}$; HRMS-EI $\mathrm{m} / z$ : calcd for $\mathrm{C}_{29} \mathrm{H}_{36} \mathrm{~N}_{5} \mathrm{O}_{7}[\mathrm{M}+\mathrm{H}]^{+}:$566.2615; found: $566.2623[\Delta-1.46$ ppm]; IR (ATR): $v=1719,1673,1621,1581,1548,1234$, $1119,953,886,806,758 \mathrm{~cm}^{-1}$.

\section{$\mathrm{N}$-(Boc-Carbamimidoyl)-1,5,7-trimethyl-2,4- dioxo-3-\{2-[3,7,8-trimethyl-2,4-dioxo-3,4- dihydrobenzo[g]pteridin-10(2H)-yl]ethyl\}-3- azabicyclo[3.3.1]nonane-7-carboxamide; Flavin-Boc-guanidin 7}

To a solution of $\mathrm{HOBt} \cdot \mathrm{H}_{2} \mathrm{O}(89 \mathrm{mg}, 580 \mu \mathrm{mol})$, EDC (90 mg, $580 \mu \mathrm{mol})$ and DIPEA $(171 \mu \mathrm{L}, 970 \mu \mathrm{mol})$ in $\mathrm{CH}_{2} \mathrm{Cl}_{2}(6.5 \mathrm{~mL})$ were added compound $6(252 \mathrm{mg}, 480 \mu \mathrm{mol})$ and mono Bocprotected guanidine $(86 \mathrm{mg}, 530 \mu \mathrm{mol})$ at $0{ }^{\circ} \mathrm{C}$. The mixture was stirred at room temperature for $20 \mathrm{~h}$, diluted with $\mathrm{CHCl}_{3}$ $(150 \mathrm{~mL})$ and washed with brine twice. The organic phase was separated, dried over magnesium sulfate and the solvents were evaporated to yield an orange solid. The crude product was purified by flash column chromatography $\left(\mathrm{CHCl}_{3}: \mathrm{MeOH}-\right.$ 50:1). Yield: $186 \mathrm{mg}, 280 \mu \mathrm{mol}, 58 \%$, orange solid; $R_{\mathrm{f}}=0.15$ $\left(\mathrm{CHCl}_{3}: \mathrm{MeOH}-50: 1\right)$; mp 255-259 ${ }^{\circ} \mathrm{C}$ (decomp.); ${ }^{1} \mathrm{H}$ NMR $\left(\mathrm{CDCl}_{3}\right) \delta=0.91-1.05\left(\mathrm{~m}, 12 \mathrm{H}, 3 \times \mathrm{CH}_{3}+3 \times \mathrm{H}_{a x}\right), 1.48(\mathrm{~s}, 9$ $\left.\mathrm{H}, \mathrm{Boc}-\mathrm{CH}_{3}\right), 2.39$ (s, $\left.3 \mathrm{H}, \mathrm{Ar}-\mathrm{CH}_{3}\right), 2.48\left(\mathrm{~s}, 3 \mathrm{H}, \mathrm{Ar}-\mathrm{CH}_{3}\right)$, 2.64-2.69 (m, $\left.3 \mathrm{H}, H_{e q}\right), 3.48\left(\mathrm{~s}, 3 \mathrm{H}, \mathrm{N}-\mathrm{CH}_{3}\right), 3.98(\operatorname{tr}, J=4.53$ $\mathrm{Hz}, 2 \mathrm{H}, \mathrm{CH}_{2}$ ), 4.84 (br s, $2 \mathrm{H}, \mathrm{CH}_{2}$ ), 7.30 (s, $1 \mathrm{H}, \mathrm{Ar}-\mathrm{H}$ ) 7.99 (s, $1 \mathrm{H}, \mathrm{Ar}-H$ ), 8.33 (br s, $1 \mathrm{H}, \mathrm{N}-H), 8.85$ (br s, $1 \mathrm{H}, \mathrm{N}-H) ;{ }^{13} \mathrm{C}$ NMR $\left(\mathrm{CDCl}_{3}\right) \delta=19.5\left(\mathrm{Ar}-\mathrm{CH}_{3}\right) 21.9\left(\mathrm{Ar}-\mathrm{CH}_{3}\right), 25.5(2 \times$ $\left.\mathrm{CH}_{3}\right), 28.1\left(\mathrm{Boc}-\mathrm{CH}_{3}\right), 28.8\left(\mathrm{~N}-\mathrm{CH}_{3}\right), 31.3\left(\mathrm{CH}_{3}\right), 37.2$ $\left(\mathrm{N}-\mathrm{CH}_{2}\right), 40.1\left(2 \times C_{q u}\right), 42.3\left(\mathrm{CH}_{2}\right), 43.3\left(\mathrm{CH}_{2}\right), 44.1$ $\left(\mathrm{N}-\mathrm{CH}_{2}\right), 44.5\left(C_{q u}\right), 83.8$ (Boc- $\left.C_{q u}\right), 115.1(C 9), 131.9(C 9 a)$, 132.8 (C6), 134.7 (C5a), 135.8 (C4a), 136.3 (C7), 146.8 (C8), $149.3(C 10 a), 153.0(\mathrm{NHCO}), 156.0$ (C2), 158.7 (Boc-CO), $160.2(C 4), 177.5$ (NHCO); ES-MS $m / z(\%): 681.4\left[\mathrm{M}+\mathrm{NH}_{4}\right]^{+}$,

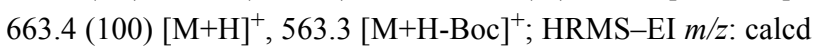
for $\mathrm{C}_{33} \mathrm{H}_{43} \mathrm{~N}_{8} \mathrm{O}_{7}[\mathrm{M}+\mathrm{H}]^{+}:$: 663.3255; found: 663.3242 [ 1.92 ppm] IR (ATR): $v=1716,1668,1634,1584,1543,1455,1367$, $1327,1236,1144,968,756 \mathrm{~cm}^{-1}$.

\section{$\mathrm{N}$-(Boc-Carbamimidoyl)-3-\{2-[10-(2-meth-} oxyethyl)-7,8-dimethyl-2,4-dioxobenzo[g]pteridin-3(2H,4H,10H)-yl]ethyl\}-1,5,7-trimethyl-

\section{2,4-dioxo-3-azabicyclo[3.3.1]nonane-7-}

\section{carboxamide; Flavin-Boc-guanidin 10}

To a solution of $\mathrm{HOBt} \cdot \mathrm{H}_{2} \mathrm{O}(226 \mathrm{mg}, 1.67 \mathrm{mmol})$, EDC (226 $\mathrm{mg}, 1.45 \mathrm{mmol})$ and DIPEA $(498 \mu \mathrm{L}, 970 \mu \mathrm{mol})$ in $\mathrm{CHCl}_{3}(10$ $\mathrm{mL})$ was added compound $9(548 \mathrm{mg}, 969 \mu \mathrm{mol})$ and mono Boc-protected guanidine $(231 \mathrm{mg}, 1.45 \mathrm{mmol})$ at $0{ }^{\circ} \mathrm{C}$. The mixture was stirred at room temperature for $20 \mathrm{~h}$, diluted with $\mathrm{CHCl}_{3}(250 \mathrm{~mL})$ and washed with water and brine. The organic phase was separated, dried over magnesium sulfate and the solvents were evaporated. The crude brown product was purified by flash column chromatography $\left(\mathrm{CHCl}_{3}: \mathrm{MeOH}: \mathrm{N}(\mathrm{Et})_{3}-\right.$ 70:1:1). Yield: $564 \mathrm{mg}, 798 \mu \mathrm{mol}, 82 \%$, yellow solid; $R_{\mathrm{f}}=0.1$ ( $\mathrm{CHCl}_{3}: \mathrm{MeOH}: \mathrm{TEA}$ - 50:1:1); mp 229-231 ${ }^{\circ} \mathrm{C}$ (decomp.); ${ }^{1} \mathrm{H}$ $\operatorname{NMR}\left(\mathrm{CDCl}_{3}\right) \delta=0.93-1.21\left(\mathrm{~m}, 12 \mathrm{H}, 3 \times \mathrm{CH}_{3}+3 \times \mathrm{H}_{a x}\right)$, $1.46(\mathrm{~s}, 9 \mathrm{H}$, Boc-CH$), 2.15\left(\mathrm{~d}, J=12.90 \mathrm{~Hz}, 1 \mathrm{H}, H_{e q}\right), 2.38$ (s, $\left.3 \mathrm{H}, \mathrm{Ar}-\mathrm{CH}_{3}\right), 2.48$ (s, $\left.3 \mathrm{H}, \mathrm{Ar}-\mathrm{CH}_{3}\right), 2.68(\mathrm{~d}, J=13.44 \mathrm{~Hz}$, $\left.2 \mathrm{H}, H_{e q}\right), 3.22\left(\mathrm{~s}, 3 \mathrm{H}, \mathrm{O}-\mathrm{CH}_{3}\right), 3.80-3.84\left(\mathrm{~m}, 4 \mathrm{H}, 2 \times \mathrm{CH}_{2}\right)$, 4.20-4.22 (m, $\left.2 \mathrm{H}, \mathrm{CH}_{2}\right), 4.79\left(\operatorname{tr}, J=5.08 \mathrm{~Hz}, 2 \mathrm{H}, \mathrm{CH}_{2}\right), 7.58$ (s, $1 \mathrm{H}, \operatorname{Ar}-H) 7.94$ (s, $1 \mathrm{H}, \mathrm{Ar}-H), 8.27$ (br s, $1 \mathrm{H}, \mathrm{N}-H), 8.75$ (br s, $1 \mathrm{H}, \mathrm{N}-H) ;{ }^{13} \mathrm{C} \mathrm{NMR}\left(\mathrm{CDCl}_{3}\right) \delta=19.5\left(\mathrm{Ar}-\mathrm{CH}_{3}\right) 21.5$ $\left(\mathrm{Ar}-\mathrm{CH}_{3}\right), 25.5\left(2 \times \mathrm{CH}_{3}\right), 28.1\left(\mathrm{Boc}-\mathrm{CH}_{3}\right), 31.3\left(\mathrm{CH}_{3}\right), 38.4$ $\left(\mathrm{N}-\mathrm{CH}_{2}\right), 40.2\left(2 \times \mathrm{C}_{q u}\right), 40.8\left(\mathrm{~N}-\mathrm{CH}_{2}\right), 43.2\left(\mathrm{~N}-\mathrm{CH}_{2}\right), 44.2(2$ $\left.\times \mathrm{CH}_{2}\right), 44.6\left(\mathrm{C}_{q u}\right), 45.2\left(\mathrm{CH}_{2}\right), 59.2\left(\mathrm{O}-\mathrm{CH}_{3}\right), 69.6\left(\mathrm{O}-\mathrm{CH}_{2}\right)$, 83.4 (Boc- $C_{q u}$ ), 116.6 (C9), 132.2 (C9a), 132.2 (C6), 134.9 (C5a), 135.6 (C4a), 136.4 (C7), 147.2 (C8), 148.7 (C10a), 153.4 (NHCO), 156.1 (C2), 158.5 (Boc-CO), 160.4 (C4), 177.5 
(NHCO), $188.7\left(C_{q u}\right)$; ES-MS $m / z(\%): 707.3(100)[\mathrm{M}+\mathrm{H}]^{+}$; HRMS-EI $m / z$ : calcd for $\mathrm{C}_{35} \mathrm{H}_{47} \mathrm{~N}_{8} \mathrm{O}_{8}[\mathrm{M}+\mathrm{H}]^{+}$: 707.3517; found: $707.3531[\Delta-2.00 \mathrm{ppm}]$; IR (ATR): $v=1706,1655$, $1634,1583,1540,1457,1366,1325,1226,1146,758 \mathrm{~cm}^{-1}$.

\section{$\mathrm{N}$-Carbamimidoyl-1,5,7-trimethyl-2,4-dioxo-3- \{2-[3,7,8-trimethyl-2,4-dioxo-3,4- dihydrobenzo[g]pteridin-10(2H)-yl]ethyl\}-3- azabicyclo[3.3.1]nonane-7-carboxamide; \\ Flavin-guanidinium 1}

Compound 7 (210 mg, $317 \mu \mathrm{mol})$ was dissolved in $\mathrm{CHCl}_{3}(25$ $\mathrm{mL})$ and hydrogen chloride saturated diethyl ether $(3 \mathrm{~mL})$ was added dropwise. After stirring for $24 \mathrm{~h}$, the solution was evaporated to $5 \mathrm{~mL}$ and diethyl ether $(15 \mathrm{~mL})$ was added to precipitate the product. The mixture was cooled to $0{ }^{\circ} \mathrm{C}$ and the solid was filtered off, washed with diethyl ether and dried. Yield: 157 $\mathrm{mg}, 262 \mu \mathrm{mol}, 83 \%$, orange-yellow solid; $R_{\mathrm{f}}=0.1$ $\left(\mathrm{CHCl}_{3}: \mathrm{MeOH}-10: 1\right)$; mp 320-322 ${ }^{\circ} \mathrm{C}$ (decomp.); ${ }^{1} \mathrm{H}$ NMR $($ DMSO-d 6$) \delta=0.91\left(\mathrm{~s}, 6 \mathrm{H}, 2 \times \mathrm{CH}_{3}\right), 1.16\left(\mathrm{~s}, 3 \mathrm{H}, \mathrm{CH}_{3}\right)$, 1.28-1.31 (m, $4 \mathrm{H}, 3 \times \mathrm{CH}_{a x}$ and $\left.\mathrm{CH}_{e q}\right), 2.41\left(\mathrm{Ar}-\mathrm{CH}_{3}\right), 2.47$ (d, $\left.J=14.39 \mathrm{~Hz}, 2 \mathrm{H}, H_{e q}\right), 2.50\left(\mathrm{Ar}^{-} \mathrm{CH}_{3}\right.$, hidden by DMSO), $3.28\left(\mathrm{~s}, 3 \mathrm{H}, \mathrm{N}-\mathrm{CH}_{3}\right), 3.90\left(\operatorname{tr}, J=5.01 \mathrm{~Hz}, 2 \mathrm{H}, \mathrm{CH}_{2}\right), 4.84(\mathrm{~s}$, $\left.2 \mathrm{H}, \mathrm{CH}_{2}\right), 7.74(\mathrm{Ar}-H), 7.97(\mathrm{Ar}-H), 8.36-8.44(\mathrm{~m}, 4 \mathrm{H}, \mathrm{NH})$, $11.38(\mathrm{~s}, 1 \mathrm{H}, \mathrm{N} H \mathrm{CO}) ;{ }^{13} \mathrm{C}$ NMR $\left(\mathrm{DMSO}-d_{6}\right) \delta=18.7$ (Ar$\left.\mathrm{CH}_{3}\right) 21.0\left(\mathrm{Ar}-\mathrm{CH}_{3}\right), 24.7\left(2 \times \mathrm{CH}_{3}\right), 28.0\left(\mathrm{~N}-\mathrm{CH}_{3}\right), 29.0$ $\left(\mathrm{CH}_{3}\right), 36.8\left(\mathrm{CH}_{2}\right), 40.1\left(\mathrm{C}_{q u}\right), 40.7\left(\mathrm{CH}_{2}\right), 42.0\left(\mathrm{CH}_{2}+\mathrm{C}_{q u}\right)$, $43.6\left(\mathrm{CH}_{2}\right), 116.0(C 9), 131.1$ (C5a), 131.3 (C6), $134.0(C 9 a)$, 135.5 (C4a), 136.2 (C7), 146.8 (C8), 149.3 (C10a), 154.9 (C2), $155.0\left(C_{q u}\right), 159.4(C 4), 176.0(C O), 177.3(C O)$; ES-MS m/z (\%): 563.3 (100) $[\mathrm{M}+\mathrm{H}]^{+}$; HRMS-EI $\mathrm{m} / \mathrm{z}$ : calcd for $\mathrm{C}_{28} \mathrm{H}_{35} \mathrm{~N}_{8} \mathrm{O}_{5}[\mathrm{M}+\mathrm{H}]^{+}:$563.2730; found: $563.2746[\Delta-2.77$ ppm]; IR (ATR): $v=1700,1643,1584,1546,1452,1306$, 1238, 1190, 1127, 1098, 1048, $753 \mathrm{~cm}^{-1}$; UV/Vis (MeCN): $\lambda_{\max }(\varepsilon)=272$ (41500), 343 (9130), 447 nm (11060); Fluorescence $(\mathrm{MeCN}): \lambda_{\max }($ emission$)=507 \mathrm{~nm}$ (excitation: $\left.445 \mathrm{~nm}\right)$.

\section{$\mathrm{N}-$ Carbamimidoyl-3-\{2-[10-(2-methoxyethyl)-} 7,8-dimethyl-2,4-dioxobenzo[g]pteridin$3(2 \mathrm{H}, 4 \mathrm{H}, 10 \mathrm{H})$-yl]ethyl\}-1,5,7-trimethyl-2,4dioxo-3-azabicyclo[3.3.1]nonane-7-carboxamide; Flavin-guanidinium 2

Compound 10 (493 mg, $698 \mu \mathrm{mol}$ ) was dissolved in $\mathrm{CHCl}_{3}(50$ $\mathrm{mL})$ and hydrogen chloride saturated diethyl ether $(6 \mathrm{~mL})$ was added dropwise. After stirring for $24 \mathrm{~h}$, the solution was evaporated to $5 \mathrm{~mL}$ and diethyl ether $(25 \mathrm{~mL})$ was added to precipitate the product. The mixture was cooled to $0{ }^{\circ} \mathrm{C}$ and the solid was filtered off, washed with diethyl ether and dried. Yield: 402 $\mathrm{mg}, 625 \mu \mathrm{mol}, 90 \%$, yellow solid; $R_{\mathrm{f}}=0.15\left(\mathrm{CHCl}_{3}: \mathrm{MeOH}-\right.$ 10:1); mp $245-247{ }^{\circ} \mathrm{C}$ (decomp.); ${ }^{1} \mathrm{H}$ NMR (DMSO- $\left.d_{6}\right) \delta=$ $0.99\left(\mathrm{~s}, 6 \mathrm{H}, 2 \times \mathrm{CH}_{3}\right), 1.17\left(\mathrm{~s}, 3 \mathrm{H}, \mathrm{CH}_{3}\right), 1.30(\mathrm{~d}, J=14.39$ $\left.\mathrm{Hz}, 2 \mathrm{H}, 2 \times \mathrm{CH}_{a x}\right), 1.38\left(\mathrm{~d}, J=12.59 \mathrm{~Hz}, 1 \mathrm{H}, \mathrm{C} H_{a x}\right), 1.84(\mathrm{~d}$, $\left.J=12.59 \mathrm{~Hz}, \mathrm{H}, \mathrm{CH}_{e q}\right), 2.39\left(\mathrm{~s}, 3 \mathrm{H}, \mathrm{Ar}-\mathrm{CH}_{3}\right), 2.51(\mathrm{~s}, 3 \mathrm{H}$, $\left.\mathrm{Ar}-\mathrm{CH}_{3}\right), 2.52\left(\mathrm{~d}, J=14.39 \mathrm{~Hz}, 2 \mathrm{H}, 2 \times \mathrm{CH}_{\mathrm{ax}}\right), 3.21(\mathrm{~s}, 3 \mathrm{H}$, O-CH $\left.H_{3}\right), 3.73-3.75\left(\mathrm{~m}, 4 \mathrm{H}, 2 \times \mathrm{CH}_{2}\right), 4.02-4.04(\mathrm{~m}, 2 \mathrm{H}$, $\left.\mathrm{CH}_{2}\right), 4.82\left(\operatorname{tr}, J=5.52 \mathrm{~Hz}, 2 \mathrm{H}, \mathrm{CH}_{2}\right), 7.89(\mathrm{Ar}-H), 7.95$ (Ar$H), 8.34-8.43$ (m, $4 \mathrm{H}, \mathrm{N} H), 11.42$ (s, $1 \mathrm{H}, \mathrm{NHCO}) ;{ }^{13} \mathrm{C} \mathrm{NMR}$ $\left(\mathrm{DMSO}-d_{6}\right) \delta=18.8\left(\mathrm{Ar}-\mathrm{CH}_{3}\right) 20.7\left(\mathrm{Ar}-\mathrm{CH}_{3}\right), 24.9\left(2 \times \mathrm{CH}_{3}\right)$, $28.6\left(\mathrm{CH}_{3}\right), 37.3\left(\mathrm{CH}_{2}\right), 39.5\left(\mathrm{CH}_{2}\right), 40.1\left(\mathrm{C}_{q u}\right), 41.2\left(\mathrm{CH}_{2}\right)$, $42.1\left(2 \times \mathrm{CH}_{2}\right), 43.8\left(\mathrm{C}_{q u}\right), 44.0\left(\mathrm{CH}_{2}\right), 116.9(\mathrm{C9}), 130.9(\mathrm{Cb})$, 131.4 (C5a), 134.1 (C9a), 135.5 (C4a), 136.3 (C7), 147.0 (C8), $148.5(C 10 a), 154.9(C 2), 155.1\left(C_{q u}\right), 159.7(C 4), 176.0(C O)$, $177.3(\mathrm{CO})$; ES-MS m/z (\%): $607.3(100)[\mathrm{M}+\mathrm{H}]^{+}$; HRMS-EI $m / z$ : calcd for $\mathrm{C}_{30} \mathrm{H}_{39} \mathrm{~N}_{8} \mathrm{O}_{6}[\mathrm{M}]^{+}: 607.2993$; found: 607.2981 [ $\Delta 1.90 \mathrm{ppm}]$; IR (ATR): $v=1692,1669,1579,1545,1456$, $1328,1235,1173,747 \mathrm{~cm}^{-1}$; UV/Vis $(\mathrm{MeCN}): \lambda_{\max }(\varepsilon)=275$ (96000), 344 (8760), $445 \mathrm{~nm}$ (10510); Fluorescence (MeCN): $\lambda_{\max }($ emission $)=509 \mathrm{~nm}($ excitation: $445 \mathrm{~nm})$.

\section{Supporting Information}

Photocatalytic experiments, UV/Vis and fluorescence spectra of $1-3$, calculated gas phase conformations, ${ }^{1} \mathrm{H}$ and ${ }^{13} \mathrm{C}$ NMR spectra of $\mathbf{1}, \mathbf{2}, \mathbf{6}, \mathbf{7}, \mathbf{9}$, and $\mathbf{1 0}$.

\section{Supporting Information File 1}

Koenig_Supporting Information

[http://www.beilstein-journals.org/bjoc/content/

supplementary/1860-5397-5-26-S1]

\section{Supporting Information File 2}

CIF file of compound $\mathbf{1}$.

[http://www.beilstein-journals.org/bjoc/content/ supplementary/1860-5397-5-26-S2]

\section{Supporting Information File 3 CIF file of compound 6.}

[http://www.beilstein-journals.org/bjoc/content/ supplementary/1860-5397-5-26-S3]

\section{Supporting Information File 4 \\ CIF file of compound 9.}

[http://www.beilstein-journals.org/bjoc/content/ supplementary/1860-5397-5-26-S4]

\section{Acknowledgments}

This work was supported by the Deutsche Forschungsgemeinschaft (GRK 640 fellowship to H.S.) and the Evonik foundation (fellowship to $\mathrm{MB}$ ). 


\section{References}

1. Müller, F., Ed. Chemistry and Biochemistry of Flavoenzymes; CRC Boca Raton, 1991.

2. Fritz, B. J.; Kusai, S.; Matsui, K. Photochem. Photobiol. 1987, 45, 113-117. doi:10.1111/j.1751-1097.1987.tb08411.x

3. Bowd, A.; Bysom, P.; Hudson, J. B.; Turnbull, J. H. Photochem. Photobiol. 1968, 8, 1-10. doi:10.1111/j.1751-1097.1968.tb05839.x

4. Niemz, A.; Rotello, V. M. Acc. Chem. Res. 1999, 32, 44-52. doi:10.1021/ar980046I

5. Gishla, S.; Massey, V. Eur. J. Biochem. 1989, 181, 1-17. doi:10.1111/j.1432-1033.1989.tb14688.x

6. Schmaderer, H.; Svoboda, J.; König, B. Flavin Photocatalysts with Substrate Binding Sites. In Activating Unreactive Substrates: The Role of Secondary Interactions; Bolm, C.; Hahn, E., Eds.; Wiley-VCH: Weinheim, 2009.

7. Mansoorabadi, S. O.; Thibodeaux, C. J.; Liu, H. J. Org. Chem. 2007, 72, 6329-6342. doi:10.1021/j00703092

8. Hemmerich, P. Prog. Natl. Prod. Chem. 1976, 33, 451-525.

9. Massey, V. Biochem. Soc. Trans. 2000, 28, 283-296. doi:10.1042/0300-5127:0280283

10. Moonen, M. J. H.; Fraaije, M. W.; Rietjens, I. M. C. M.; Laane, C.; van Berkel, W. J. H. Adv. Synth. Catal. 2002, 344, 1023-1035. doi:10.1002/1615-4169(200212)344:10<1023::AID-ADSC1023>3.0.CO ;2-T

11. Murahashi, S.-I.; Ono, S.; Imada, Y. Angew. Chem. 2002, 114, 2472-2474.

Angew. Chem., Int. Ed. 2002, 41, 2366-2368. doi:10.1002/1521-3773(20020703)41:13<2366::AID-ANIE2366>3.0.CO ;2-S.

12. Gelelcha, F. G. Chem. Rev. 2007, 107, 3338-3361. doi:10.1021/cr0505223

13. Fitzpatrick, P. F. Acc. Chem. Res. 2001, 34, 299-307. doi:10.1021/ar0000511

14. Wiest, O.; Harrison, C. B.; Saettel, N. J.; Cibulka, R.; Sax, M.; König, B. J. Org. Chem. 2004, 69, 8183-8185. doi:10.1021/jo0494329

15. Julliard, M.; Chanon, M. Chem. Rev. 1983, 83, 425-506. doi:10.1021/cr00056a003

16. Murahashi, S.-I.; Oda, T.; Masui, Y. J. Am. Chem. Soc. 1989, 111, 5002-5003. doi:10.1021/ja00195a076

17. Ball, S.; Bruice, T. C. J. Am. Chem. Soc. 1980, 102, 6498-6503. doi:10.1021/ja00541a019

18. Jonsson, S. Y.; Färnegårdh, K.; Bäckvall, J.-E. J. Am. Chem. Soc. 2001, 123, 1365-1371. doi:10.1021/ja0035809

19. Lindén, A. A.; Johansson, M.; Hermanns, N.; Bäckvall, J.-E. J. Org. Chem. 2006, 71, 3849-3853. doi:10.1021/jo060274q

20. Cibulka, R.; Vasold, R.; König, B. Chem.-Eur. J. 2004, 10, 6223-6231. doi:10.1002/chem.200400232

21. Svoboda, J.; Schmaderer, H.; König, B. Chem. Eur. J. 2008, 14, 1854-1865. doi:10.1002/chem.200701319

22. Imada, Y.; lida, H.; Ono, S.; Murahashi, S.-I. J. Am. Chem. Soc. 2003, 125, 2868-2869. doi:10.1021/ja028276p

23. Behrens, C.; Ober, M.; Carell, T. Eur. J. Org. Chem. 2002, 3281-3289. doi:10.1002/1099-0690(200210)2002:19<3281::AID-EJOC3281>3.0.C O;2-I

24. Legrand, Y.-M.; Gray, M.; Cooke, G.; Rotello, V. M. J. Am. Chem. Soc. 2003, 125, 15789-15795. doi:10.1021/ja036940b

25. Lindén, A. A.; Hermanns, N.; Ott, S.; Krüger, L.; Bäckvall, J.-E. Chem.-Eur. J. 2005, 11, 112-119. doi:10.1002/chem.200400540

26. Bergstad, K.; Bäckvall, J.-E. J. Org. Chem. 1998, 63, 6650-6655. doi:10.1021/jo980926d
27. Smit, C.; Fraaije, M. W.; Minnaard, A. J. J. Org. Chem. 2008, 73, 9482-9485. doi:10.1021/jo801588d

28. Bruice, T. C. Acc. Chem. Res. 1980, 13, 256-262. doi:10.1021/ar50152a002

29. Piera, J.; Bäckvall, J.-E. Angew. Chem. 2008, 120, 3558-3576. Angew. Chem., Int. Ed., 2008, 47, 3506-3523. doi:10.1002/anie.200700604.

30. Hollmann, F.; Taglieber, A.; Schulz, F.; Reetz, M. T. Angew. Chem. 2007, 119, 2961-2964.

Angew. Chem., Int. Ed., 2007, 46, 2903-2906. doi:10.1002/anie.200605169.

31. Kemp, D. S.; Petrakis, K. S. J. Org. Chem. 1981, 46, 5140-5143. doi:10.1021/jo00338a014

32. Bach, T.; Bergmann, H.; Grosch, B.; Harms, K.; Herdtweck, E. Synthesis 2001, 1395-1405. doi:10.1055/s-2001-15231

33. Grosch, B.; Orlebar, C. N.; Herdtweck, E.; Massa, W.; Bach, T. Angew. Chem. 2003, 32, 3693-3696.

Angew. Chem., Int. Ed. 2003, 32, 3693-3696. doi:10.1002/anie.200351567.

34. Bauer, A.; Westkämper, F.; Grimme, S.; Bach, T. Nature 2005, 436, 1139-1140. doi:10.1038/nature03955

35. Svoboda, J.; König, B. Chem. Rev. 2006, 106, 5413-5430. doi:10.1021/cr050568w

36. Breitenlechner, S.; Bach, T. Angew. Chem. 2008, 120, 8075-8077. Angew. Chem., Int. Ed. 2008, 47, 7957-7959. doi:10.1002/anie.200802479.

37. Mori, K.; Murai, O.; Hashimoto, S.; Nakamura, Y. Tetrahedron Lett. 1996, 37, 8523-8526. doi:10.1016/0040-4039(96)01981-8

38. Amirsakis, D. G.; Elizarov, A. M.; Garcia-Garibay, M. A.; Glink, P. T.; Stoddart, J. F.; White, A. J. P.; Williams, D. J. Angew. Chem. 2003, 115, 1158-1164.

Angew. Chem., Int. Ed. 2003, 42, 1126-1132. doi:10.1002/anie.200390296.

39. Bach, T.; Bergmann, H.; Harms, K. J. Am. Chem. Soc. 1999, 121 , 10650-10651. doi:10.1021/ja992209m

40. Bach, T.; Bergmann, H.; Brummerhop, H.; Lewis, W.; Harms, K. Chem.-Eur. J. 2001, 7, 4512-4521. doi:10.1002/1521-3765(20011015)7:20<4512::AID-CHEM4512>3.0.C $\mathrm{O} ; 2-\mathrm{H}$

41. Bach, T.; Bergmann, H.; Harms, K. Angew. Chem. 2000, 112, 2391-2393.

Angew. Chem., Int. Ed. 2000, 39, 2302-2304. doi:10.1002/1521-3773(20000703)39:13<2302::AID-ANIE2302>3.3.CO ;2-Y.

42. Bach, T.; Bergmann, H.; Grosch, B.; Harms, K. J. Am. Chem. Soc. 2002, 124, 7982-7990. doi:10.1021/ja0122288

43. Selig, P.; Bach, T. J. Org. Chem. 2006, 71, 5662-5673. doi:10.1021/jo0606608

44. Marcus, R. A. Angew. Chem. 1993, 105, 1161-1172. Angew. Chem., Int. Ed. 1993, 32, 1111-1121. doi:10.1002/anie.199311113.

45. Bailey, P. J.; Pace, S. Coord. Chem. Rev. 2001, 214, 91-141. doi:10.1016/S0010-8545(00)00389-1

46. Schug, K. A.; Lindner, W. Chem. Rev. 2005, 105, 67-113. doi:10.1021/cr040603j

47. Best, M. D.; Tobey, S. L.; Anslyn, E. V. Coord. Chem. Rev. 2003, 240, 3-15.

48. Bloudeau, P.; Segura, M.; Pérez-Fernández, R.; de Mendoza, J. Chem. Soc. Rev. 2007, 36, 198-210. doi:10.1039/b603089k 
49. Schmuck, C. Coord. Chem. Rev. 2006, 250, 3053-3067. doi:10.1016/j.ccr.2006.04.001

50. Schmaderer, H.; Hohenleutner, A.; König, B. Synth. Commun. 2009. In print.

51. Rebek, J., Jr.; Askew, B.; Kolloran, M.; Nemeth, D.; Lin, F.-T. J. Am. Chem. Soc. 1987, 109, 2426-2431. doi:10.1021/ja00242a029

52. Askew, B.; Ballester, P.; Buhr, C.; Jeong, K.-S.; Jones, S.; Parris, K.; Williams, K.; Rebek, J., Jr. J. Am. Chem. Soc. 1989, 111, 1082-1090. doi:10.1021/ja00185a044

53. Semi-empirical AM1, Spartan program package.

54. Levy, R. M.; Gallicchio, E. Annu. Rev. Phys. Chem. 1998, 49, 531-567. doi:10.1146/annurev.physchem.49.1.531

55. Davis, M. E.; McCammon, J. A. Chem. Rev. 1990, 90, 509-521. doi:10.1021/cr00101a005

56. Rozanska, X.; Chipot, C. J. Chem. Phys. 2000, 112, 9691-9694.

57. Luo, R.; David, L.; Hung, H.; Devany, J.; Gilson, M. K. J. Phys. Chem. B 1999, 103, 727-736. doi:10.1021/jp982715i

58. Matsunov, A.; Lazaridis, T. J. Am. Chem. Soc. 2003, 125, 1722-1730. doi:10.1021/ja025521w

59. Fagnoni, M.; Dondi, D.; Ravelli, D.; Albini, A. Chem. Rev. 2007, 107, 2725-2756. doi:10.1021/cr068352x

60. Stuhlmann, F.; Jäschke, A. J. Am. Chem. Soc. 2002, 124, 3238-3244. doi:10.1021/ja0167405

61. Helm, M.; Petermeier, M.; Ge, B.; Fiammengo, R.; Jäschke, A. J. Am. Chem. Soc. 2005, 127, 10492-10493. doi:10.1021/ja052886i

62. Fukuzumi, S.; Yuasa, J.; Miyagawa, T.; Suenobu, T. J. Phys. Chem. A 2005, 109, 3174-3181. doi:10.1021/jp050347u

63. Fukuzumi, S.; Okamoto, T.; Ohkubo, K. J. Phys. Chem. A 2003, 107, 5412-5418. doi:10.1021/jp034080f

64. Sun, D.; Hubig, S. M.; Kochi, J. K. Photochem. Photobiol. A: Chem. 1999, 122, 87-94. doi:10.1016/S1010-6030(99)00007-6

\section{License and Terms}

This is an Open Access article under the terms of the Creative Commons Attribution License (http://creativecommons.org/licenses/by/2.0), which permits unrestricted use, distribution, and reproduction in any medium, provided the original work is properly cited.

The license is subject to the Beilstein Journal of Organic Chemistry terms and conditions:

(http://www.beilstein-journals.org/bjoc)

The definitive version of this article is the electronic one which can be found at: doi:10.3762/bjoc.5.26 